\title{
Estudio del grado de endurecimiento y resistencia al desgaste por deslizamiento del acero AISI 1045 endurecido por temple con refrigerante automotor y para mecanizado Study of hardening and wear resistance of the AISI 1045 steel quenched with automotive and machining coolant
}

\author{
Joan Ravagli-Reyes ${ }^{1 a}$, Eduardo Pérez-Ruiz ${ }^{1 b}$, Jorge Llano-Martínez ${ }^{1 c}$ \\ ${ }^{1}$ Grupo de investigación D+TEC, programa de Ingeniería Mecánica, Universidad de Ibagué, Colombia. \\ Correos electrónicos: ${ }^{a} 2120132016 @$ estudiantesunibague.edu.co, b eduardo.perez@unibague.edu.co, \\ c jorge.llano@unibague.edu.co
}

Recibido: 30 junio, 2018. Aceptado: 28 enero, 2019. Versión final: 13 febrero, 2019.

\begin{abstract}
Resumen
Para mejorar las propiedades mecánicas de los aceros, se utiliza el tratamiento térmico de temple. De esta forma se incrementa la dureza, la resistencia a la tracción y, por consiguiente, se espera que también la resistencia al desgaste. Este trabajo se enfoca en estudiar la resistencia al desgaste del acero AISI 1045 templado en agua, en refrigerante para motor de vehículo y en refrigerante para mecanizado. Los temples fueron realizados en discos de 76,2 $\mathrm{mm}$ de diámetro y $8 \mathrm{~mm}$ de espesor. Los temples se realizaron en un horno eléctrico a temperatura de austenización de $870{ }^{\circ} \mathrm{C}$ y tiempo de sostenimiento de 50 minutos. Los medios de enfriamientos utilizados fueron agua, refrigerante automotor Quallitor, $\mathrm{y}$ tres mezclas de refrigerante para mecanizado, en este caso Solubeg, (10\% refrigerante, $90 \%$ agua, $30 \%$ refrigerante, $70 \%$ agua, y $50 \%$ refrigerante, $50 \%$ agua). Los resultados obtenidos indican que en todos los aceros templados el coeficiente de fricción fue inferior, en comparación con el disco en estado comercial. Igualmente se evidenció que la tasa de desgaste fue alta en los aceros de mayor dureza. Finalmente, se encontró que aceite de mecanizado en mezcla con agua permite obtener dureza intermedia, coeficiente de fricción intermedio y baja pérdida de masa.
\end{abstract}

Palabras clave: Acero AISI 1045; medios de temple; resistencia al desgaste.

\begin{abstract}
In order to improve the mechanical properties of the steels, the quenching heat treatment is used; in this way, the hardness, the tensile strength and consequently, the wear resistance are increased. This work focuses on studying the wear resistance of AISI 1045 steel quenched in water, in coolant for vehicle engine and in coolant for machining. The samples were made in $76.2 \mathrm{~mm}$ diameter and $8 \mathrm{~mm}$ thick discs. The quenchings were carried out in an electric furnace at an austenitizing temperature of $870^{\circ} \mathrm{C}$ and a holding time of 50 minutes. The cooling media used was water, Quallitor automotive coolant and three coolant mixtures for machining, in this case, Solubeg, (10\% coolant - $90 \%$ water, $30 \%$ coolant $-70 \%$ water and $50 \%$ coolant $-50 \%$ water ). The results indicated that in all quenched steels the coefficient of friction was lower compared with the material in commercial state. It was also evident that the wear rate was high in the harder steels. Finally, it was evidenced that machining oil mixed with water allows obtaining intermediate hardness, intermediate coefficient of friction and low mass loss.
\end{abstract}

Keywords: AISI 1045 steel; quenching media; wear resistance.

ISSN impreso: 1657 - 4583. ISSN en línea: 2145 - 8456, CC BY-ND 4.0 cc) (i) $€$

J. Ravagli-Reyes, E. Pérez-Ruiz, J. Llano-Martínez, "Estudio del grado de endurecimiento y resistencia al desgaste por deslizamiento del acero AISI 1045 endurecido por temple con refrigerante automotriz y para mecanizado," Rev. UIS Ing., vol. 18, no. 2, pp. 113-118, 2019. doi: 10.18273/revuin.v18n2-2019010 


\section{Introducción}

El acero AISI 1045 es un acero con un nivel medio de resistencia mecánica, ampliamente utilizado en la construcción de partes de máquinas que requieran dureza y tenacidad. Sin embargo, para aprovechamiento de las propiedades mecánicas, este acero puede ser endurecido a través de tratamientos térmicos. En particular, el tratamiento térmico de temple tiene como objetivo endurecer y aumentar la resistencia de los aceros, lo que eleva el acero a una temperatura ligeramente superior a la de austenización y luego enfría rápidamente el material, mediante un medio conveniente, como agua, aceite, agua con sales, etc. El medio de enfriamiento es uno de los factores más importante durante el tratamiento de temple, y es así como se ha identificado que el agua salada agitada favorece la obtención de altas durezas, mientras que los aceites, durezas intermedias [1-3].

Durante el enfriamiento del material, se atraviesa por tres etapas distintas. La primera etapa ocurre al introducir el acero en el líquido; el choque térmico genera una capa de vapor que rodea el material y genera el enfriamiento por conducción y radiación a través de la capa gaseosa. Esta primera etapa depende directamente del punto de ebullición y la temperatura del líquido. La segunda etapa es transición entre el vapor y el líquido que rodea la pieza; al disminuir la temperatura del material el vapor se va separando de la superficie y es reemplazado por líquido, lo que hace más rápido el enfriamiento. En la tercera etapa, el acero se encuentra a una temperatura más baja que la de ebullición del líquido, de manera que el enfriamiento es realizado por el líquido, y esta transferencia de calor es dada a través de conducción y convección. Esta etapa es las más lenta de las tres, y depende directamente de la conductividad térmica del líquido y la diferencia de temperaturas entre ambos [1]. Esta secuencia de etapas es la responsable de las propiedades mecánicas finalmente obtenidas, por lo que se demuestra la importancia que tiene la selección de la sustancia de temple [2,3]. En este sentido, en diferentes investigaciones se ha explorado el uso de sustancias de temple diferentes al agua o a los aceites minerales para temple [4-6], así como las propiedades termodinámicas de las sustancias durante el temple del acero [7].

Es así como Criollo [8] exploró el temple del acero AISI 1045 utilizando agua, aceite sintético SAE 10W30, aceite de maíz, aceite de oliva, aceite de girasol, manteca vegetal y aceite de palma. Con los resultados se ratificó que el agua es la sustancia que genera mayor dureza en el acero; alcanza $55 \mathrm{HRC}$ en la superficie y $53 \mathrm{HRc}$ en el núcleo. En cuanto a las sustancias no tradicionales, como es el caso de los aceites vegetales, se determinó que el mejor resultado se obtiene con el aceite de Oliva, y se obtiene un valor de dureza de $51 \mathrm{HRC}$ en la superficie y 48 HRC en el núcleo, seguido del aceite de palma, con 50HRC en la superficie y 48 HRC en el núcleo.

En cuanto a la exploración de sustancias de temple alternativas, Ndaliman [9] estableció que el acero templado con agua alcanzó las mejores propiedades en resistencia y dureza, mientras que el acero templado con aceite de palma tiene alta tenacidad. Otros autores han centrado sus investigaciones en analizar el comportamiento del medio de enfriamiento. Souza et al. [10] lograron obtener las curvas de enfriamiento para distintos aceites vegetales (soya, maíz, algodón, canola, coco, girasol) y su posible uso como medios de enfriamiento, lo que determina que los aceites vegetales presentan una baja estabilidad térmica ante la oxidación, así como alta viscosidad cinemática, a diferencia de los derivados del petróleo. En esta misma línea de búsqueda de nuevas sustancias, Pérez et al. [11] analizaron el uso de sustancias refrigerantes con aplicaciones en automotores y en procesos de mecanizado industrial. Los resultados muestran que el uso del refrigerante para radiadores favorece la obtención de alta dureza superficial. El uso de refrigerante para mecanizado en mezcla con agua permitió obtener valores de dureza inferiores a los alcanzados con agua o con el refrigerante de radiador; sin embargo, a nivel microestructural fueron obtenidas microestructuras del tipo Bainita, las que aportan dureza y alta tenacidad, lo que sugiere un buen equilibrio en comportamiento mecánico.

Existen otras formas de lograr endurecimiento en el acero AISI 1045. Charadia et al. [12] evalúan el grado de endurecimiento y resistencia al degaste del acero mediante nitruración iónica y posterior temple por inducción. Los resultados indicaron que los mayores valores de dureza, así como los menores valores de coeficiente de fricción y de pérdida de material fueron alcanzados en la condición de nitruración y temple.

El principal objetivo de esta investigación se centra en identificar el grado de endurecimiento y resistencia al desgaste que presenta el acero AISI 1045 al ser templado en sustancias refrigerantes no tradicionales para el endurecimiento de los aceros.

\section{Procedimiento}

\subsection{Material y tratamientos térmicos}

El material utilizado para este estudio fue acero en estado comercial AISI 1045, cortado en discos de 3in de diámetro $(76,2 \mathrm{~mm})$, con un espesor de $8 \mathrm{~mm}$, pulidos con papel abrasivo de $\mathrm{SiC}$ número 80,180 y 220 , y se obtuvo 
una rugosidad promedio de $\mathrm{Ra}=0,20 \mu \mathrm{m}$ (Figura 1 ). Se realizaron seis tratamientos térmicos de temple y uno de revenido.

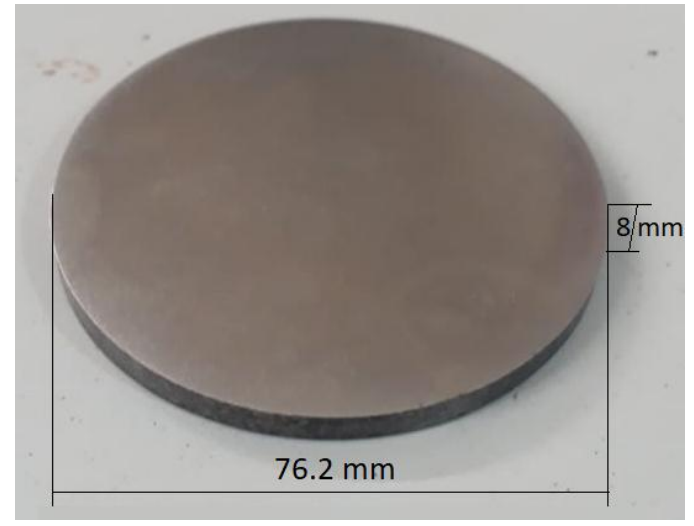

Figura 1. Geometría de los cuerpos de ensayo de acero AISI 1045.

En la tabla 1 se presentan los medios de enfriamiento y las condiciones de temple utilizadas.

Tabla 1. Parámetros de los tratamientos de temple en el acero AISI 1045.

\begin{tabular}{|c|c|c|c|c|}
\hline $\begin{array}{c}\text { Tratamiento } \\
\text { termico }\end{array}$ & $\begin{array}{c}\text { Medio de } \\
\text { enfriamiento }\end{array}$ & $\begin{array}{r}\text { Temperatura } \\
\text { sostenimiento }\end{array}$ & $\begin{array}{c}\text { Tiempo de } \\
\text { subida }\end{array}$ & $\begin{array}{c}\text { Tiempo de } \\
\text { sostenimiento }\end{array}$ \\
\hline Temple & Agua & \multirow{5}{*}{$870^{\circ} \mathrm{C}$} & \multirow{5}{*}{30 minutos } & \multirow{5}{*}{50 minutos } \\
\hline Temple & Quallitor & & & \\
\hline Temple & $\begin{array}{l}\text { Mezcla Solubeg } \\
10 \% \text { - Agua } 90 \% \\
\end{array}$ & & & \\
\hline Temple & $\begin{array}{l}\text { Mezcla Solubeg } \\
30 \% \text { - Agua } 70 \%\end{array}$ & & & \\
\hline Temple & $\begin{array}{l}\text { Mezcla Solubeg } \\
50 \% \text { - Agua } 50 \%\end{array}$ & & & \\
\hline $\begin{array}{l}\text { Temple / } \\
\text { Revenido } \\
\end{array}$ & Agua / Al aire & $870^{\circ} \mathrm{C} / 400^{\circ} \mathrm{C}$ & $\begin{array}{c}30 \mathrm{~min} / \\
20 \mathrm{~min}\end{array}$ & $50 \mathrm{~min} / 40 \mathrm{~min}$ \\
\hline
\end{tabular}

\subsection{Dureza y desgaste}

Para la medición de la dureza se realizaron 13 indentaciones a lo largo del diámetro de cada disco. El reporte de dureza se realiza en escala Rockwell C. Para esto fue utilizado un durómetro TIME HBRVU 187,5. El ensayo de desgaste se realizó en condición en seco, en un equipo Ball-on-Disk, mediante metodología de la norma ASTM G99 [13]. Para esto fue utilizada una esfera de óxido de zirconio ( $\mathrm{ZrO} 2)$ utilizada en rodamientos, aplicando una carga normal de $50 \mathrm{~N}$ contra el disco de acero, el cual giró a una velocidad lineal constante de $0,342 \mathrm{~m} / \mathrm{s}$, a lo largo de $1500 \mathrm{~m}$ de distancia de desgaste. La pérdida de masa que sufrió cada disco fue medida a través de una balanza analítica PRECISA XB 220A con precisión de 0,0001g.

\subsection{Obtención microestructural}

Para la identificación de la microestructura fue realizado un proceso metalográfico. Las muestras fueron atacadas químicamente con una solución concentrada al $2 \%$ de Nital, para luego ir al microscopio y obtener la microestructura de cada muestra, tomando imágenes con 20X y 50X aumentos para el disco en estado comercial, mientras que, para los discos templados, a 50X y $100 \mathrm{X}$ aumentos. Para la obtención de estas imágenes se utilizó el microscopio OLYMPUS BX51RF.

\section{Resultados y discusión}

\subsection{Dureza y desgaste}

Comparando los promedios de durezas obtenidos plasmados en la Tabla 2, se identifica que la dureza del acero AISI 1045 sin tratamiento (COM) fue el valor más bajo, con una magnitud de 17 HRC. De igual manera la dureza del disco templado en agua (AGU) es la de mayor magnitud, con un valor de 59,5 $\mathrm{HRC}$, mientras que al aplicar el tratamiento térmico de revenido luego del temple en agua (TYR) se disminuye la dureza a 38,5 HRC. Al templar con refrigerante automotor como el Quallitor (QUA), se alcanzaron valores de dureza altos (58,1 HRC promedio), y se comportó casi de igual manera que la dureza del temple en agua.

Tabla 2. Valores de dureza HRC alcanzados después de los tratamientos.

\begin{tabular}{|c|c|}
\hline MUESTRA & DUREZA PROMEDIO (HRC) \\
\hline COM & 17 \\
\hline AGU & 59,5 \\
\hline QUA & 58,1 \\
\hline S10 & 55 \\
\hline S 30 & 37,2 \\
\hline S50 & 31,4 \\
\hline TYR & 38,5 \\
\hline
\end{tabular}

Al utilizar el refrigerante para mecanizado Solubeg en diferentes concentraciones, se identifica que la mezcla de menor concentración de Solubeg (S10) causa un valor alto de dureza (55 HRC). Mientras que, al aumentar la concentración del refrigerante para mecanizado, disminuye indirectamente la dureza, al utilizar una mezcla de $50 \%$ Solubeg y $50 \%$ agua (S50), permite obtener una dureza promedio de 31,4 HRC.

La figura 2 presenta los coeficientes de fricción para cada muestra en función de la distancia de deslizamiento. Es 
posible identificar que el coeficiente de fricción más alto se obtuvo en el disco sin tratamiento (COM). De igual manera se observa que se obtuvo un coeficiente intermedio para los discos bajo condiciones de temple y revenido (TYR), temple en mezcla de Solubeg al $30 \%$
(S30) y $50 \%$ (S50). Finalmente, se identifica que para los discos bajo condiciones de temple en agua (AGU), Quallitor (QUA) y mezcla Solubeg al $10 \%$ (S10), se obtuvo el coeficiente de fricción más bajo.

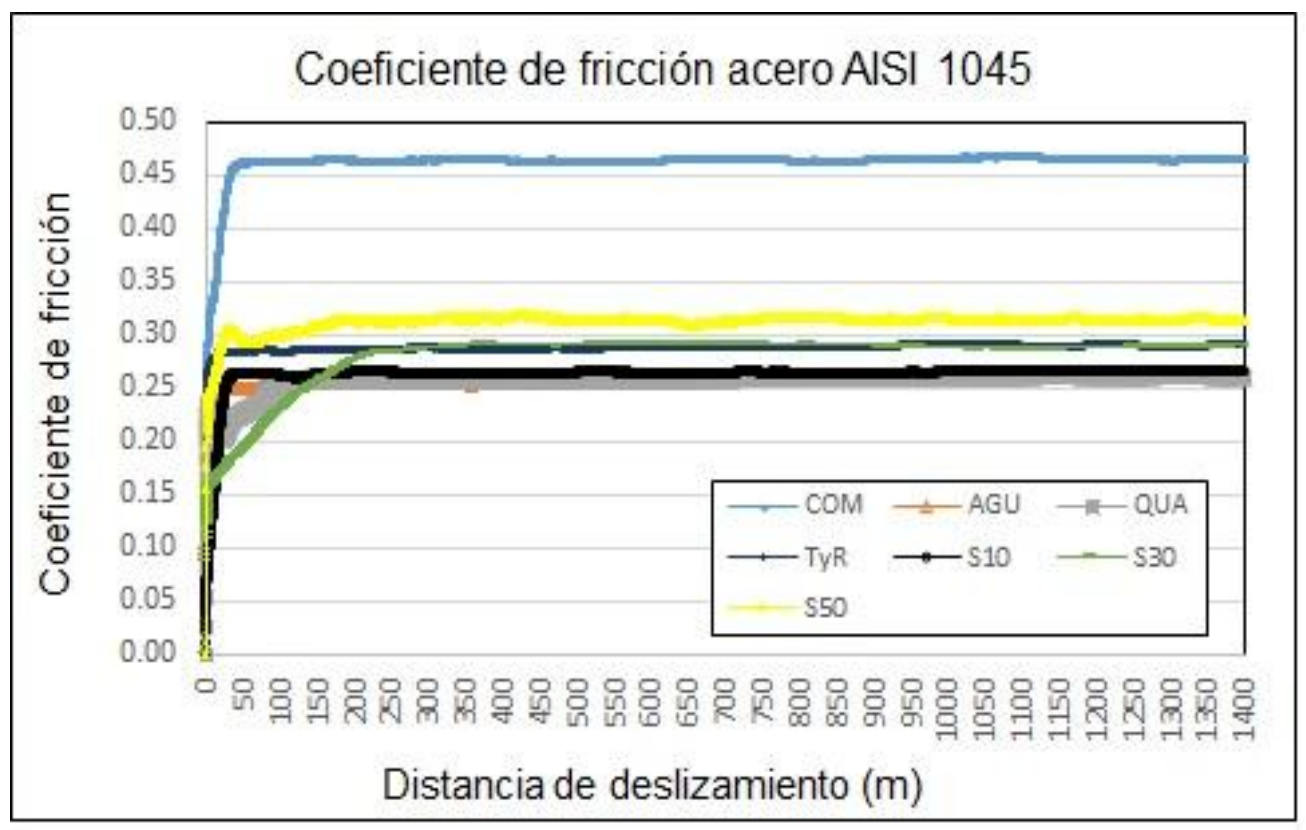

Figura 2. Comportamiento del coeficiente de fricción en cada caso estudiado.

En la tabla 3 se presenta la pérdida de masa en promedio para cada condición de ensayo estudiada. En la tabla se evidencia que la mayor pérdida de masa se obtuvo en los discos en las condiciones de temple en agua y en Quallitor, mientras que la menor pérdida de masa se evidenció en los discos templados en mezclas de refrigerante para mecanizado. Se lograron obtener pérdidas de masa intermedias para los discos templados en mezcla de $10 \%$ refrigerante, $90 \%$ agua, $30 \%$ refrigerante, $70 \%$ agua, y en el disco templado en agua y revenido

Tabla 3. Pérdida de masa de las muestras después del ensayo de desgaste Ball-On-Disk.

\begin{tabular}{|c|c|}
\hline MUESTRA & PERDIDADE MASA(g) \\
\hline COM & 0,0553 \\
\hline AGU & 0,1536 \\
\hline QUA & 0,1420 \\
\hline S10 & 0,0980 \\
\hline S30 & 0,0804 \\
\hline S50 & 0,0557 \\
\hline TYR & 0,0823 \\
\hline
\end{tabular}

\subsection{Análisis microestructural}

Las microestructuras obtenidas se resumen en las figuras 3 y 4 . En las figuras 3 a y 3 b se observa la microestructura del acero AISI 1045 sin tratamiento (COM). En esta condición, la microestructura obtenida muestra principalmente perlita con ferrita proeutectoide. Las figuras $3 \mathrm{c}$ y $3 \mathrm{~d}$ muestran la microestructura del acero AISI 1045 templado en agua, y se manifiesta una estructura en forma de agujas, de la que resulta esta estructura martensita, obtenida durante el enfriamiento rápido. Las figuras $3 \mathrm{e}$ y $3 \mathrm{f}$ corresponden al disco templado en Quallitor. En estas imágenes se observa martensita de agujas largas.

En la figura 4 se presentan las imágenes obtenidas para el refrigerante en diferentes mezclas de agua. En las figuras $4 \mathrm{a}$ y $4 \mathrm{~b}$ se muestran martensita $\mathrm{y}$ pequeños contornos de grano de perlita. En las figuras 4c y $4 d$ se observa mayor concentración de troostita (perlita muy fina) en los contornos de grano, así como bainita superior. En las figuras $4 \mathrm{e}$ y $4 \mathrm{f}$, al igual que en las imágenes $4 \mathrm{c}$ y $4 \mathrm{~d}$, se observa perlita fina en los contornos de los granos, así como bainita en el interior de estos contornos. 
Estudio del grado de endurecimiento y resistencia al desgaste por deslizamiento del acero AISI 1045 endurecido por temple con refrigerante automotriz y para mecanizado

De acuerdo con la literatura, una alta dureza superficial en los materiales favorece la reducción del coeficiente de fricción, lo cual se ratifica con los resultados de la tabla 2 y la figura 2 , en donde se establece que el temple en agua y en Quallitor permitió la obtención de coeficientes de fricción de 0,25 en promedio. En estado comercial el valor del coeficiente de fricción alcanzado fue de 0,47 aproximadamente.

De la misma forma, es altamente conocido que una de las formas de reducir la pérdida de material (desgaste) en los metales es justamente el aumento en la dureza superficial; sin embargo, es posible que el aumento de dureza lleve al surgimiento de fenómenos de desgaste similares [14]. En este estudio se observa que la mayor pérdida de material se presenta en los casos de temple con agua y temple en Quallitor, es decir, en aquellos casos en los cuales la dureza fue la mayor. Una forma de explicar dicho comportamiento se debe a que el agua y el Quallitor, por su baja viscosidad, favorecen una alta tasa de enfriamiento, y producen una alta tasa de transformación de martensita, como se evidencia en las figuras 3 c y $3 d$. Esta alta densidad de martensita origina concentración de esfuerzos residuales, y, por tanto, un comportamiento más frágil del material, razón por la cual es altamente recomendable la realización de tratamientos de revenido.

En cuanto al uso de las mezclas del lubricante para mecanizado, los resultados indican que el coeficiente de fricción para estos casos no es tan alto como el que presenta el acero sin ningún tratamiento, e intenta estar más cerca de valores bajos obtenidos en el temple en agua y en Quallitor. Para estos casos ese valor estuvo aproximadamente en 0,28. En cuanto a la pérdida de masa, se puede establecer que estas mezclas bajan la tasa de desgaste. Esto se debe a que, por presentar mayores viscosidades que el agua, estas sustancias reducen un poco la capacidad de evacuación de calor, favorecen el surgimiento de bainita y perlita fina en la microestructura, lo que lleva a la obtención de durezas intermedias y gran tenacidad.

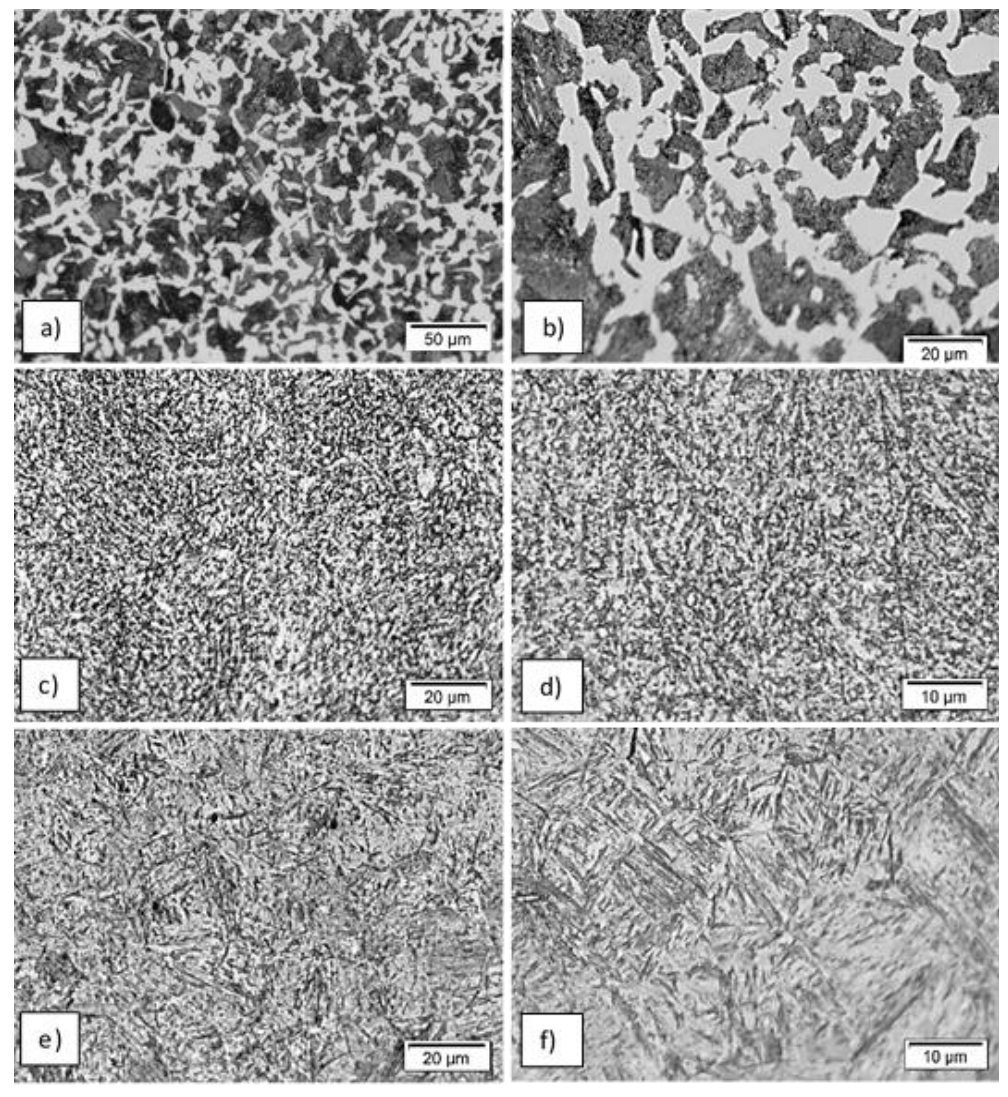

Figura 3. Microestructuras del acero AISI 1045. a) Sin tratamiento 20X, b) sin tratamiento 50X y c) templado en agua 50X, d) templado en agua 100X, e) templado en Quallitor 50X, f) templado en Quallitor 100X. 


\section{Conclusiones}

Los resultados obtenidos en este trabajo permiten analizar y demostrar cómo influyen los medios de enfriamiento usados en el temple, en propiedades y comportamiento como dureza, coeficiente de fricción y pérdida de masa. En el caso del uso de refrigerante automotor, como sustancia para temple de aceros, es posible establecer que genera el mismo efecto que el temple en agua, en cuanto al alto valor de dureza, así como favorece obtener bajo valor del coeficiente de fricción. Así como un alto valor de la pérdida de masa. En cuanto al uso de mezclas de refrigerante para mecanizado es posible concluir que en proporciones de $30-50 \%$ de aceite y su equivalencia 70-50\% en agua, estas sustancias favorecen obtener durezas intermedias 35HRC, así como reducir el coeficiente de fricción a valores de aproximadamente 0,3 junto con reducir el desgaste.

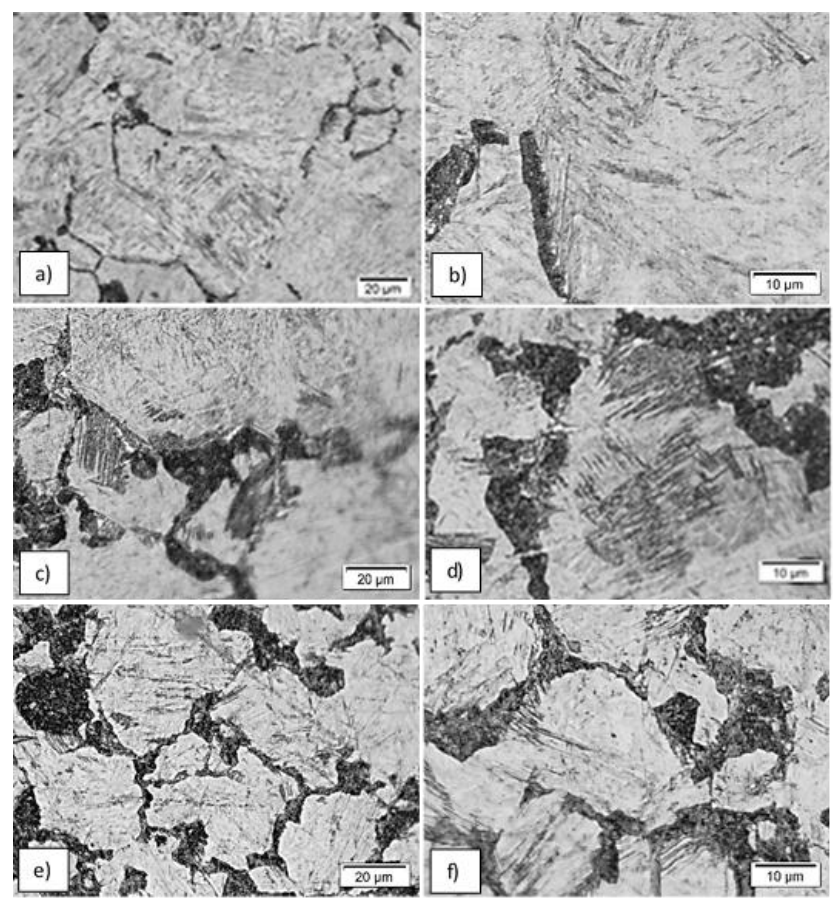

Figura 4. Microestructuras del acero AISI 1045 templado en las diferentes mezclas de refrigerante para mecanizado. a) $10 \%$ refrigerante, $90 \%$ agua $50 \mathrm{X}$, b) $10 \%$ refrigerante, $90 \%$ agua $100 \mathrm{X}$, c) $30 \%$ refrigerante, $70 \%$ agua $50 \mathrm{X}$, d) $30 \%$ refrigerante, $70 \%$ agua $100 \mathrm{X}$, e) $50 \%$ refrigerante, $50 \%$ agua $50 \mathrm{X}$, f) $50 \%$ refrigerante, $50 \%$ agua $100 \mathrm{X}$.

\section{Agradecimientos}

Los autores expresan su gratitud a la Universidad de Ibagué por apoyar financiera y técnicamente el proyecto de investigación n. ${ }^{\circ}$ 17-460-INT.

\section{Referencias}

[1] J. Apraiz Barreiro, "Tratamientos térmicos de los aceros", Madrid: s.l. Inversiones editoriales dossat-2000, 2002.
[2] N. kobasko., "Fffect of accuracy of temperature measurement on determination of heat transfer coefficient during quenching in liquid media", Journal of ASTM International, vol. 9, no.2, pp. 1-12, 2011.

[3] M. Aronov, N. Kobasko, J. Powell, "Practical applications of intensive quenching methods", proc. 5th IASME/WSEAS int. Conference on Heat Transfer, Thermal Engineering and Environment, Athens Greece, 2007, pp. 160-166.

[4] R. Otero, L .Canale, , and G. Totten, "Use of vegetable oils and animal oils as steel quenchants: A 
Estudio del grado de endurecimiento y resistencia al desgaste por deslizamiento del acero AISI 1045

endurecido por temple con refrigerante automotriz y para mecanizado

historical review-1850-2010", Journal of ASTM International, vol. 9, pp.1-38, 2012

[5] C. Civera,, B. Rivolta, R. Simencio-Otero, G. Lucio, G. Totten and L. Canale, "Vegetable oils as quenchants for steel: residual stresses dimensional changes", Materials performance and characterization, 2014, pp. 1-21. doi: 10.1520/MPC20140039

[6] M.B Adeyemi,.., and S,M Adedayo,.., "Vegetable oils as quenchants for hardening médium carbon Steel", Journal of applied science and technology, vol. 14, pp. 74-78, 2009,

[7] E. García, I. Amaya, R. Correa, "Algoritmos de optimización en la estimación de propiedades termodinámicas en tiempo real durante el tratamiento térmico de materiales con microondas", Rev. UIS Ing., vol. 16, no. 2, pp. 129-140, 2017. doi: https://doi.org/10.18273/revuin.v16n2-2017012

[8] M. X Criollo-Camacho, "Estudio del temple en el acero utilizando grasas", Universidad Técnica de Ambato, Ambato-Ecuador, 2011.

[9] M. B. Ndaliman, "An assessment of mechanical properties of medium carbon steel under different quenching media", A.U. Journal of Technology, vol. 10, no. 2, pp. 100-104, 2006.

[10] E. C.de Souza, , O. Friedel, , F. Luiz,., G. Totten, , L. Canale, "Quenching and heat transfer properties of aged and unaged vegetable oils", Journal of petroleum science research, vol. 2, no. 1, pp. 41-47, 2013.

[11] E. A. Pérez-Ruiz, A. Galeano, L. Negrín Hernández, "Evaluación de la dureza y microestructura del acero 1045 templado en sustancias refrigerantes no tradicionales", Scientia et Technica, vol. 21, no. 3, pp. 213-218, 2016.

[12] R. Charadia, M. Regis, E. alibon, S. P. Brühl,. "Comportamiento al desgaste de un acero SAE 1045, templado por inducción posterior a la nitruración iónica", en $15^{\circ}$ Congreso Internacional de Metalurgias y Materiales, Concepción, Chile, 2015.

[13] "Standard test method for wear testing with a pinon-disk apparatus”, ASTM G-99, 2010.

[14] M. Vodă, A. Pertuz-Comas, V. A Şerban, "Correlation between mass loss on the cavitation erosion and the fatigue stress level for amartensitic stainless steel," Rev. UIS Ing., vol. 18, no. 1, pp. 11-20, 2019. doi: 10.18273/revuin.v18n1-2019001 